\title{
Morphological homology, evolution, and proposed nomenclature for bear dentition
}

Qigao Jiangzuo, Jinyi Liu, and Jin Chen

Acta Palaeontologica Polonica 64 (4), 2019: 693-710 doi:https://doi.org/10.4202/app.00629.2019

Bears are a group of carnivores with diverse diets and complicated dental structure. Several large rearrangements of dental structures are known in different lineages of bears, making the homology of dental structures between the different bears difficult to evaluate. By tracing the evolutionary processes of the two lineages of bears with the most complicated dental structure, i.e., the giant panda lineage (Ailuropodinae) and cave bear lineage (Ursinae), we were able to clarify the homology of dental structures of the two subfamilies. We define a new assemblage of dental nomenclature (based mainly on the homology to the giant panda) that can be very useful to infer the evolution of fossil bears. The evolutionary positions of some fossil bears are reviewed based on our results.

Key words: Mammalia, Ailuropodinae, Ursinae, dentition, homology, Pleistocene, China.

Qigao Jiangzuo [jiangzuo@ivpp.ac.cn] (corresponding author), Key Laboratory of Vertebrate Evolution and Human Origins of Chinese Academy of Sciences; Institute of Vertebrate Paleontology and Paleoanthropology, Chinese Academy of Sciences and Center for Excellence in Life and Paleoenvironment, Beijing, 100044, China; University of Chinese Academy of Sciences, Beijing, 100049, China; Division of Paleontology, American Museum of Natural History, New York, 10024, USA. Jinyi Liu [liujinyi@ivpp.ac.cn] (corresponding author), Key Laboratory of Vertebrate Evolution and Human Origins of Chinese Academy of Sciences; Institute of Vertebrate Paleontology and Paleoanthropology, Chinese Academy of Sciences and Center for Excellence in Life and Paleoenvironment, Beijing, 100044, China. Jin Chen [chenjin@ivpp.ac.cn], Key Laboratory of Vertebrate Evolution and Human Origins of Chinese Academy of Sciences; Institute of Vertebrate Paleontology and Paleoanthropology, Chinese Academy of Sciences, Beijing, 100044, China.

This is an open-access article distributed under the terms of the Creative Commons 
Attribution License (for details please see creativecommons.org), which permits unrestricted use, distribution, and reproduction in any medium, provided the original author and source are credited.

PoFif Full text $(817.0 \mathrm{kB})$ 